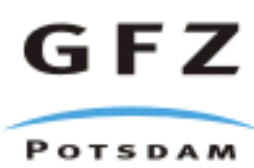

Originally published as:

Jahn, S. (2008): High-pressure phase transitions in MgSiO3 orthoenstatite studied by atomistic computer simulation. - American Mineralogist, 93, 4, 528-532

DOI: 10.2138/am.2008.2710. 


\title{
High pressure phase transitions in $\mathrm{MgSiO}_{3}$ orthoenstatite studied by atomistic computer simulation
}

\author{
Sandro Jahn \\ GeoForschungsZentrum Potsdam, Department 4, Telegrafenberg, 14473 Potsdam, \\ Germany. E-mail: jahn@gfz-potsdam.de
}

\begin{abstract}
Molecular dynamics simulations and first principles electronic structure calculations are used to study the structural behavior of orthoenstatite, $\mathrm{MgSiO}_{3}$, at high pressures. The calculations suggest two possible high-pressure polymorphs of orthoenstatite, one with $\mathrm{P} 2{ }_{1} \mathrm{ca}$ and the other with Pbca symmetry. Both polymorphs are structurally related to orthoenstatite. Molecular dynamics simulations reveal the displacive nature of the phase transitions between the three phases. Electronic structure calculations predict a phase transition from orthoenstatite to the metastable $\mathrm{P} 2{ }_{1}$ ca structure at $9 \mathrm{GPa}$, which may explain the anomalies in elastic and vibrational properties observed experimentally. A second metastable transition from the $\mathrm{P} 2{ }_{1}$ ca to the high-pressure Pbca structure may be observable above $20 \mathrm{GPa}$.
\end{abstract}

Keywords: DFT, MD simulation, enstatite, phase transition, high pressure 


\section{Introduction}

Pyroxenes, (Mg,Fe)SiO 3 , are important constituents of the Earth's crust and upper mantle (Ringwood 1975; Anderson 1989). A detailed understanding of their phase relations as a function of pressure and temperature is required to model the properties and the dynamics of the Earth's upper mantle and transition zone. The phase diagram of the Mg end member, $\mathrm{MgSiO}_{3}$, includes a number of polymorphic structures with a pyroxene structure, which is based on chains of edge-sharing $\mathrm{MgO}_{6}$ octahedra and corner-sharing $\mathrm{SiO}_{4}$ tetrahedra. The different polymorphs differ in the stacking sequence of the oxygen layers, which in the "ideal pyroxenes" are close packed (Thompson and Downs 2003).

The ground state structure at ambient conditions is monoclinic low-clinoenstatite (LCEn) with space group $\mathrm{P} 22_{1} / \mathrm{c}$ (Presnall 1995). A displacive phase transition at high pressure leads to the monoclinic high-pressure clinoenstatite (HP-CEn) with space group C2/c (Angel et al. 1992). Besides the monoclinic phases, two polymorphs with orthorhombic symmetry are well known, orthoenstatite (OEn, space group Pbca) and protoenstatite (PEn, space group Pbcn). While the latter is a high temperature phase in the $\mathrm{MgSiO}_{3}$ system, OEn may exist as a metastable phase at ambient conditions. This is due to the reconstructive nature of the phase transition between OEn and any of the CEn structures, which requires reorientation of the stacked layers (Coe and Kirby 1975; Jahn and Martoňák 2007).

Recent experimental studies suggest the existence of metastable, unquenchable phases that should be structurally related to either PEn or OEn. Yang et al. (1999) reported a structural transformation in $\left(\mathrm{Mg}_{1.54} \mathrm{Li}_{0.23} \mathrm{Sc}_{0.23}\right) \mathrm{Si}_{2} \mathrm{O}_{6}$ protopyroxene from a phase with 
space group Pbcn to a phase with space group $\mathrm{P} 2{ }_{1} \mathrm{cn}$ at pressures between 2.03 and 2.50 GPa. Early molecular dynamics simulations proposed a high temperature PEn phase with space group Bmcm just below the melting point (Matsui and Price 1992). Jackson et al. (2004) predicted a new high temperature phase of OEn with space group Cmca from the softening of the elastic moduli. Such a high temperature OEn phase was observed in recent molecular dynamics simulations (Miyake et al. 2004), but with no change in symmetry.

Furthermore, a high pressure phase transition in OEn was observed in several experimental studies. Kung et al. (2004) observed a substantial softening of the bulk modulus above $10 \mathrm{GPa}$ and before the intended transition from OEn to HP-CEn. The existence of a metastable phase was also deduced from Raman and x-ray diffraction data (Lin 2003; Lin et al. 2005). The reversibility of the transition suggests a displacive mechanism, which excludes the possibility of an OEn to HP-CEn transition. In the latter case, pressure release would lead to the LCEn phase (Angel et al. 1992). However, the structure of the high pressure OEn phase has not yet been resolved.

Here, classical molecular dynamics (MD) simulations and first principles electronic structure calculations in the framework of density functional theory (DFT) are used to study possible high pressure phases of orthoenstatite (HP-OEn). Whereas the MD simulations are used to demonstrate the displacive nature of the phase transformations, the more accurate DFT calculations are needed to refine the structural models and to obtain reliable transition pressures.

\section{Computational details}

MD simulations are performed using an advanced ionic interaction model (AIM) (Aguado 
et al. 2003; Madden et al. 2006). The model takes into account the Coulomb interaction between charged particles, short-ranged repulsion due to the overlap of the charge densities, dispersion, ionic polarization effects and ion shape deformations. Recently, a set of AIM potentials for the Ca-Mg-Al-Si-O system was parameterized by reference to first principles electronic structure calculations. The model was shown to be accurate and transferable in a wide range of pressures, temperatures and compositions (Jahn and Madden 2007). Here, the same set of interatomic potentials is used.

MD simulation cells are chosen to contain 640 ions (128 $\mathrm{MgSiO}_{3}$ units, 1x2x4 supercell of the OEn unit cell) and a time step of $\Delta t=1 \mathrm{fs}$ is used. For direct observation of the phase transition, $\mathrm{MD}$ simulations are run at constant temperature $(T=1000 \mathrm{~K})$ in the NPT ensemble. Pressure and temperature are controlled by a Nose-Hoover thermostat coupled to a barostat (Martyna et al. 1994). During compression (decompression), the pressure is increased (decreased) with a constant rate of $1 \mathrm{GPa} / \mathrm{ps}$.

For the DFT calculations we use the ABINIT code (Gonze et al. 2002, 2005) that is based on pseudopotentials and planewaves. It relies on an efficient Fast Fourier Transform algorithm (Goedecker 1997) for the conversion of wavefunctions between real and reciprocal space, and on the adaptation to a fixed potential of the band-by-band conjugate gradient method (Payne et al. 1992). The exchange correlation functional is treated in the local density approximation (LDA) (Perdew and Zunger 1981). Optimized normconserving pseudopotentials (Rappe et al. 1990) are generated with the OPIUM code ${ }^{1}$ and a planewave energy cutoff of $1000 \mathrm{eV}$ is chosen. The Brillouin zone of the primitive lattice is sampled

\footnotetext{
${ }^{1}$ http://opium.sourceforge.net
} 
with suitable Monkhorst-Pack (MP) grid (Monkhorst and Pack 1976), i.e. a 1x2x4 MP grid for OEn. All DFT calculations are performed in the athermal limit ( $T=0 \mathrm{~K})$. For the calculation of the enthalpy curves, cell parameters and atomic positions of the different structures are optimized at various pressures.

\section{Results}

In order to validate the models, the optimized lattice parameters of OEn and LCEn at $P=0$ GPa, $T=0 \mathrm{~K}$ predicted by DFT and AIM calculations are compared to experimental data obtained at ambient conditions (Sasaki et al. 1982; Morimoto et al. 1960). Results are listed in Table 1. Considering the temperature difference (zero vs. ambient), the agreement between the models and experiment is within the expectations of modern simulation techniques, i.e. deviations are in the order of about one to two percent in the lattice constants. The systematic underestimation of the lattice constants in the DFT calculations is due to overbinding in LDA. The classical AIM potential performs well bearing in mind that it has not been specifically parameterized for enstatites (Jahn and Madden 2007).

Starting with the OEn phase, molecular dynamics simulations are performed at constant temperature, $T=1000 \mathrm{~K}$. The evolution of the simulation cell volume and the respective cell lengths $a, b$ and $c$ are shown in Figures 1 and 2. During compression, a jump in the cell parameters is observed around $21 \mathrm{GPa}$. The reverse transition takes place at $16 \mathrm{GPa}$. The volume change across the transition is about $1.6-1.7 \%$. The volume compression towards the high pressure phase is entirely along the $c$-direction (by about 3.6\%) whereas both lattice parameters $a$ and $b$ slightly increase by about one percent (Fig. 2). Investigation of the high pressure phase suggests no change in symmetry across the transition, i.e. its 
structure remains in space group Pbca.

This new phase that will be referred to as HP-OEn1 hereafter is one possible candidate for a high-pressure metastable post-OEn phase. Other candidates should also be structurally related to OEn. Crystallographic studies on ideal pyroxenes suggest the possible existence of orthorhombic phases with $\mathrm{P} 2{ }_{1}$ ca symmetry (Thompson and Downs 2003). One of them is Thompson's predicted inversion form for orthopyroxene (Thompson 1970). This structure will be referred to as HP-OEn2. Since HP-OEn2 is not observed at any pressure as a stable phase in the MD simulations, we check the structural evolution if HP-OEn2 is used as an initial structure. It turns out that at low pressures the structure immediately transforms into OEn whereas at high pressures a transition to HP-OEn1 is observed. Hence, the classical potential does not generate a (meta-) stability field for the HP-OEn2 structure.

Due to the small energy differences expected between structurally similar phases, electronic structure calculations that are generally more accurate and reliable may provide a more quantitative picture of the phase relations than classical potentials. In the following, results of the DFT calculations are presented. At a given $P$ and $T$, the structure with the lowest free energy is thermodynamically stable. In the athermal limit ( $T=0 \mathrm{~K})$, the entropic term does not contribute to the free energy and it is sufficient to compare the enthalpy $H$ of different phases, which is given by $H=U+P V$. Both the internal energy $U$ and the pressure $P$ at a given volume are calculated in the DFT calculations after performing a structural relaxation. The resulting enthalpy curves for different orthorhombic $\mathrm{MgSiO}_{3}$ polymorphs are shown in Figure 3 as a function of $P$. The respective changes in cell volumes are shown in Figure 4 together with experimental data from Angel and Hugh-Jones (1994). 
The DFT calculations predict correctly LCEn as the ground state structure at ambient pressure and low $T$. Only slightly higher in energy is OEn, which at ambient conditions can coexist with LCEn. At about 3 GPa, the enthalpy curves of HP-CEn and LCEn cross and HP-CEn becomes the thermodynamically stable phase. The transition pressure is slightly lower than that inferred from phase diagram, which gives about $5 \mathrm{GPa}$ at $T=0 \mathrm{~K}$ (Presnall 1995). The transition is accompanied by a volume change of about $3 \%$, which is consistent with experiment (Angel et al. 1992).

At low $T$, kinetic barriers may not only prevent the transition between OEn and LCEn but also between OEn and HP-CEn well above the static transition pressure. Therefore, transitions to metastable orthorhombic phases may be observed. Indeed, the two candidate HP-OEn structures, HP-OEn1 and HP-OEn2, become thermodynamically more stable than OEn at $14 \mathrm{GPa}$ and $9 \mathrm{GPa}$, respectively. HP-OEn2 with $\mathrm{P} 2{ }_{1} \mathrm{ca}$ symmetry has a lower enthalpy than HP-OEn1 up to about $20 \mathrm{GPa}$. At $P=10 \mathrm{GPa}$, the volume change between OEn and HP-OEn1 is about 2.6\%, whereas it is only about $1.3 \%$ between OEn and HPOEn2 (Fig. 4). The respective lattice parameters of OEn, HP-OEn1 and HP-OEn2 obtained from the DFT structure refinement at $10 \mathrm{GPa}$ are given in Table 2. Finally, energy dispersive x-ray patterns of the three polymorphic phases are calculated using the DFT optimized structures at 15 GPa (Fig. 5).

\section{Discussion and conclusions}

The observation of the phase transitions between the different orthoenstatite polymorphs in MD simulations at temperatures of $1000 \mathrm{~K}$ suggests low energy transition barriers and displacive transition mechanisms similar to that of the LCEn to HP-CEn transition. This is 
consistent with the experimental observation that the high pressure OEn phase is not quenchable but gradually transforms back to OEn below 9 GPa (Lin 2003; Kung et al. 2004). The failure of the MD simulation to stabilize HP-OEn2 underlines the difficulties in constructing accurate classical potentials for complex materials. Here, the combination of classical simulation with electronic structure calculations has proven a powerful approach to study the phase relations and to gain insight into the kinetics of crystalline phase transformations.

Let us now discuss the structural differences between the three orthoenstatite polymorphs. As expected for a displacive transition, the sequence of M1 sites is unchanged across the transitions. However, the three structures exhibit a different ratio of O- to Srotated $\mathrm{SiO}_{4}$ chains, where $\mathrm{S}$ - and O-rotations describe a different sense of rotation with respect to the underlying M1 site octahedra (Thompson and Downs 2003). While in OEn all chains are O-rotated, there are $50 \%$ of S-rotated chains in HP-OEn1 and $25 \%$ of Srotated chains in HP-OEn2. In all three structures, every second layer along the $a$-direction consists of O-rotated chains only. The tetrahedral rotations in the other layer are as illustrated in Figure 6. While in OEn also the other layer consists of O-rotated chains only, all chains of this layer are changed to S-rotation in HP-OEn1. Alternating S- and O-rotated chains are observed in HP-OEn2 instead. Hence, HP-OEn2 is an intermediate structure between OEn and HP-OEn1, which results in a lower symmetry structure $\left(\mathrm{P} 2{ }_{1} \mathrm{ca}\right.$ instead of Pbca). One could also argue that HP-OEn2 is the ideal pyroxene structure whereas OEn and HP-OEn1 are "related structures" (Thompson and Downs 2003). The intermediate character of HP-OEn2 is also visible in the enthalpy curves (Fig. 3) and the cell parameters (Table 2). 
In order to compare the structural changes with experimental observations, energy dispersive x-ray diffraction patterns of the three orthoenstatites are plotted in Figure 5 together with experimental data from Kung et al. (2004). For both high pressure phases, the (321) peaks merge into the (610) peak, which is in agreement with experiment (Kung et al. 2004). It also seems that in the experiment the (420) and (221) peaks are split at high $P$. This would be consistent with the HP-OEn2 phase. The most prominent difference between the patterns of the three polymorphs is in a broad feature containing the (131) and (202) peaks. Only the pattern of HP-OEn2 resembles the double peak structure observed experimentally. A strong peak at the (431) position, as predicted for the HP-OEn1 phase, is not observed. This analysis supports the preference for HP-OEn2 as the most probable high-pressure orthoenstatite phase.

In conclusion, the ideal pyroxene structure with $\mathrm{P} 2{ }_{1} \mathrm{ca}$ symmetry is the most likely metastable high pressure polymorph of orthoenstatite in the pressure range from about 9 GPa to at least $20 \mathrm{GPa}$. If the transition to HP-CEn is prevented, e.g. at low temperatures, another phase transition to the HP-OEn1 phase (space group Pbca) may be observable above $20 \mathrm{GPa}$. These predictions from atomistic simulations still need experimental confirmation.

\section{Acknowledgments}

We thank S. Speziale for helpful discussions. The helpful comments of two anonymous reviewers are acknowledged.

\section{References}


Aguado, A., Bernasconi, L., Jahn, S., and Madden, P.A. (2003) Multipoles and interaction potentials in ionic materials from planewave-DFT calculations. Faraday Discussions, 124, 171-184.

Anderson, D.L. (1989) Theory of the Earth. Blackwell, Boston.

Angel, R.J., Chopelas, A., and Ross, N.L. (1992) Stability of high-density clinoenstatite at upper-mantle pressures. Nature 358, 322-324.

Angel, R.J. and Hugh-Jones, D.A. (1994) Equations of state and thermodynamic properties of enstatites pyroxenes. Journal of Geophysical Research 99, 1977719783.

Coe, R.S. and Kirby, S.H. (1975) The orthoenstatite to clinoenstatite transformation by shearing and reversion by annealing: Mechanism and potential applications. Contributions to Mineralogy and Petrology 52, 29-55.

Goedecker, S. (1997) Fast radix 2, 3, 4 and 5 kernels for fast Fourier transformations on computers with overlapping multiply-add instructions. SIAM Journal on Scientific Computing 18, 1605-1611.

Gonze, X., Beuken, J.-M., Caracas, R., Detraux, F., Fuchs, M., Rignanese, G.-M., Sindic, L., Verstraete, M., Zerah, G., Jollet, F., Torrent, M., Roy, A., Mikami, M., Ghosez, P., Raty, J.-L., and Allan, D.C. (2002) First-principles computation of material properties : the abinit software project. Computational Materials Science 25, 478-492.

Gonze, X., Rignanese, G.-M., Verstraete, M., Beuken, J.-M., Pouillon, Y., Caracas, R., Jollet, F., Torrent, M., Zerah, G., Mikami, M., Ghosez, P., Veithen, M., Raty, J.- 
Y., Olevano, V., Bruneval, F., Reining, L., Godby, R., Onida, G., Hamann, D.R., and Allan, D.C. (2005) A brief introduction to the abinit software package. Zeitschrift für Kristallographie 220, 558-562.

Jackson, J.M., Sinogeikin, S.V., Carpenter, M.A., and Bass, J.D. (2004) Novel phase transition in orthoenstatite. American Mineralogist 89, 239-245.

Jahn, S. and Madden, P.A. (2007) Modeling Earth materials from crustal to lower mantle conditions: A transferable set of interaction potentials for the CMAS system. Physics of the Earth and Planetary Interiors 162, 129-139.

Jahn, S. and Martoňák, R. (2008) Plastic deformation of orthoenstatite and the orthoto high-pressure clinoenstatite transition: A metadynamics simulation study, Physics and Chemistry of Minerals 35, 17-23.

Kung, J., Li, B., Uchida, T., Wang, Y., Neuville, D., and Liebermann, R.C. (2004) In situ measurements of the sound velocities and densities across the orthopyroxene $\rightarrow$ high-pressure clinopyroxene transition in $\mathrm{MgSiO}_{3}$ at high pressure. Physics of the Earth and Planetary Interiors 147, 27-44.

Lin, C., Chao, J., and Lin, C. (2005) Metastable phase transition of orthoenstatite $\left(\mathrm{MgSiO}_{3}\right)$ under high pressure. Solid State Sciences 7, 293-297.

Lin, C.-C. (2003) Pressure-induced metastable phase transition in orthoenstatite $\left(\mathrm{MgSiO}_{3}\right)$ at room temperature: a Raman spectroscopic study. Journal of Solid State Chemistry 174, 403-411.

Madden, P.A., Heaton, R., Aguado, A., and Jahn, S. (2006) From first-principles to material properties. Journal of Molecular Structure (Theochem) 771, 9-18. 
Martyna, G.J., Tobias, D.J., and Klein, M.L. (1994) Constant pressure molecular dynamics algorithms. Journal of Chemical Physics 101, 4177-4189.

Matsui, M. and Price, G.D. (1992) Computer simulations of the $\mathrm{MgSiO}_{3}$ polymorphs. Physics and Chemistry of Minerals 18, 365-372.

Miyake, A., Shimobayashi, N., and Kitamura, M. (2004) Isosymmetrical structural phase transition of orthoenstatite: Molecular dynamics simulation. American Mineralogist 89, 1667-1672.

Monkhorst, H.J. and Pack, J.D. (1976) Special points for Brillouin-zone integrations. Physical Review B 13, 5188-5192.

Morimoto, N., Appleman, D.E., and Evans, H.T. (1960) The crystal structures of clinoenstatite and pigeonite. Zeitschrift für Kristallographie 114, 120-147.

Payne, M.C., Teter, M.P., Allan, D.C., Arias, T.A., and Joannopoulos, J.D. (1992) Iterative minimization techniques for ab initio total-energy calculations: molecular dynamics and conjugate gradients. Reviews of Modern Physics 64, 1045-1097.

Perdew, J.P. and Zunger, A. (1981) Self-interaction correction to density-functional approximations for many-electron systems. Physical Review B 23, 5048-5079.

Presnall, D.C. (1995) Mineral Physics and Crystallography, A Handbook of Physical Constants. Vol. 2 of AGU Reference Shelf. American Geophysical Union, Ch. Phase diagrams of Earth-forming minerals, 248-268.

Rappe, A.M., Rabe, K.M., Kaxiras, E., and Joannopoulos, J.D. (1990) Optimized pseudopotentials. Physical Review B 41, 1227-1230.

Ringwood, A.E. (1975) Composition and Petrology of the Earth’s Mantle. McGraw- 
Hill, New York.

Sasaki, S., Takeuchi, Y., Fujino, K., and Akimoto, S. (1982) Electron-density distributions of three orthopyroxenes, $\mathrm{Mg}_{2} \mathrm{Si}_{2} \mathrm{O}_{6}, \mathrm{Co}_{2} \mathrm{Si}_{2} \mathrm{O}_{6}$, and $\mathrm{Fe}_{2} \mathrm{Si}_{2} \mathrm{O}_{2}$. Zeitschrift für Kristallographie 156, 279-297.

Thompson, J.B. (1970) Geometrical possibilities for amphibole structures: model biopyriboles. American Mineralogist 55, 292-293.

Thompson, R.M. and Downs, R.T. (2003) Model pyroxenes I: Ideal pyroxene topologies. American Mineralogist 88, 653-666.

Yang, H., Finger, L.W., Conrad, P.G., Prewitt, C.T., and Hazen, R.M. (1999) A new pyroxene structure at high pressure: Single-crystal x-ray and Raman study of the Pbcn-P $2_{1} \mathrm{cn}$ phase transition in protopyroxene. American Mineralogist 84, 245256. 


\section{Figure captions}

Figure 1: Evolution of the volume per formula unit of OEn during molecular dynamics simulations at constant $T=1000 \mathrm{~K}$. Both compression and decompression curves are shown.

Figure 2: Evolution of the unit cell parameters $a, b$ and $c$ of OEn during molecular dynamics simulations at constant $T=1000 \mathrm{~K}$. Both compression and decompression curves are shown.

Figure 3: Phase stability of different orthorhombic and monoclinic enstatite phases predicted by DFT calculations at $T=0 \mathrm{~K}$ represented by enthalpy difference curves using OEn as the reference structure. At a given $P$, the phase with the lowest enthalpy, $H$, is the thermodynamically stable phase.

Figure 4: Volume compression at $T=0 \mathrm{~K}$ of orthorhombic and monoclinic enstatite phases predicted by DFT calculations compared to experimental results at $300 \mathrm{~K}$ from Angel and Hugh-Jones (1994). The curves of OEn and LCEn are almost identical. All cell volumes from the DFT calculations are scaled by a factor of 1.044 to account for the systematic LDA error.

Figure 5: Calculated energy dispersive x-ray diffraction patterns of (a) OEn, (b) HP-OEn1 and (c) HP-OEn2 using the DFT optimized positions at $P=15 \mathrm{GPa}$ and $T=0 \mathrm{~K}$. The diffraction angle was chosen to be $5.6^{\circ}$ to obtain an energy scale similar to the experimental studies. An energy resolution of $0.5 \mathrm{keV}$ is assumed, which determines the width of the peaks. (d) Experimental data at 16.8 GPa from Kung et al. (2004) are shown for 
comparison.

Figure 6: Polyhedral representation of the structural differences between OEn, HP-OEn1 and HP-OEn2. The transition between OEn and HP-OEn2 involves the rotation of one chain from $\mathrm{O}$ to $\mathrm{S}$. In HP-OEn1, both chains are S-rotated. The layer of $\mathrm{SiO}_{4}$ chains that is not shown here remains essentially unaltered with O-rotated chains.

\section{Tables}

\begin{tabular}{|c|c|c|c|c|c|c|}
\hline & \multicolumn{3}{|l|}{ OEn } & \multicolumn{3}{|l|}{ LCEn } \\
\hline & Experiment & DFT & AIM & Experiment & DFT & AIM \\
\hline a $(\AA)$ & 18.23 & 18.06 & 17.93 & 9.63 & 9.45 & 9.47 \\
\hline $\mathrm{b}(\AA)$ & 8.82 & 8.62 & 8.81 & 8.83 & 8.67 & 8.80 \\
\hline c $(\AA)$ & 5.18 & 5.10 & 5.16 & 5.19 & 5.09 & 5.18 \\
\hline$\beta\left(^{\circ}\right)$ & & & & 108.3 & 108.0 & 108.6 \\
\hline
\end{tabular}

Table 1: Model predictions for the lattice parameters of orthoenstatite and low clinoenstatite at $P=0 \mathrm{GPa}$ and $T=0 \mathrm{~K}$ compared to experimental data at ambient conditions (Sasaki et al. 1982; Morimoto et al. 1960). 


\begin{tabular}{|l|l|l|l|}
\hline & OEn & HP-OEn1 & HP-OEn2 \\
\hline space group & Pbca & Pbca & P2 ${ }_{1}$ ca \\
\hline a (̊) & 17.68 & 17.82 & 17.75 \\
\hline b (导) & 8.431 & 8.522 & 8.469 \\
\hline c $(\AA)$ & 4.989 & 4.770 & 4.883 \\
\hline V $\left(\AA^{3}\right)$ & 743.5 & 724.3 & 733.9 \\
\hline
\end{tabular}

Table 2: Lattice parameters of OEn, HP-OEn1 and HP-OEn2 at $P=10 \mathrm{GPa}$ and $T=0 \mathrm{~K}$ from DFT calculations. Complete crystallographic information is provided as supplemental online material on the MSA website at http://www.minsocam.org. 


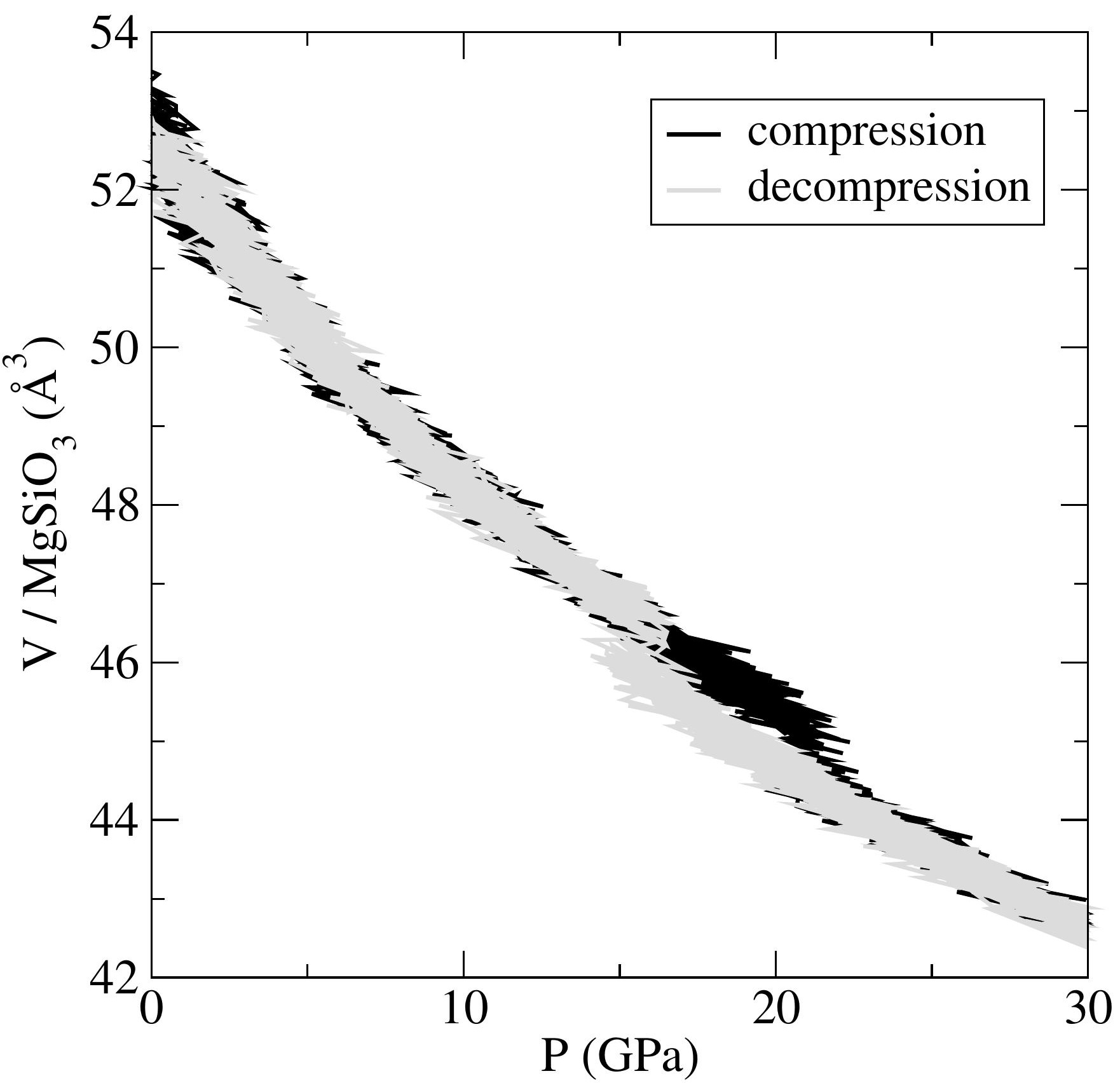




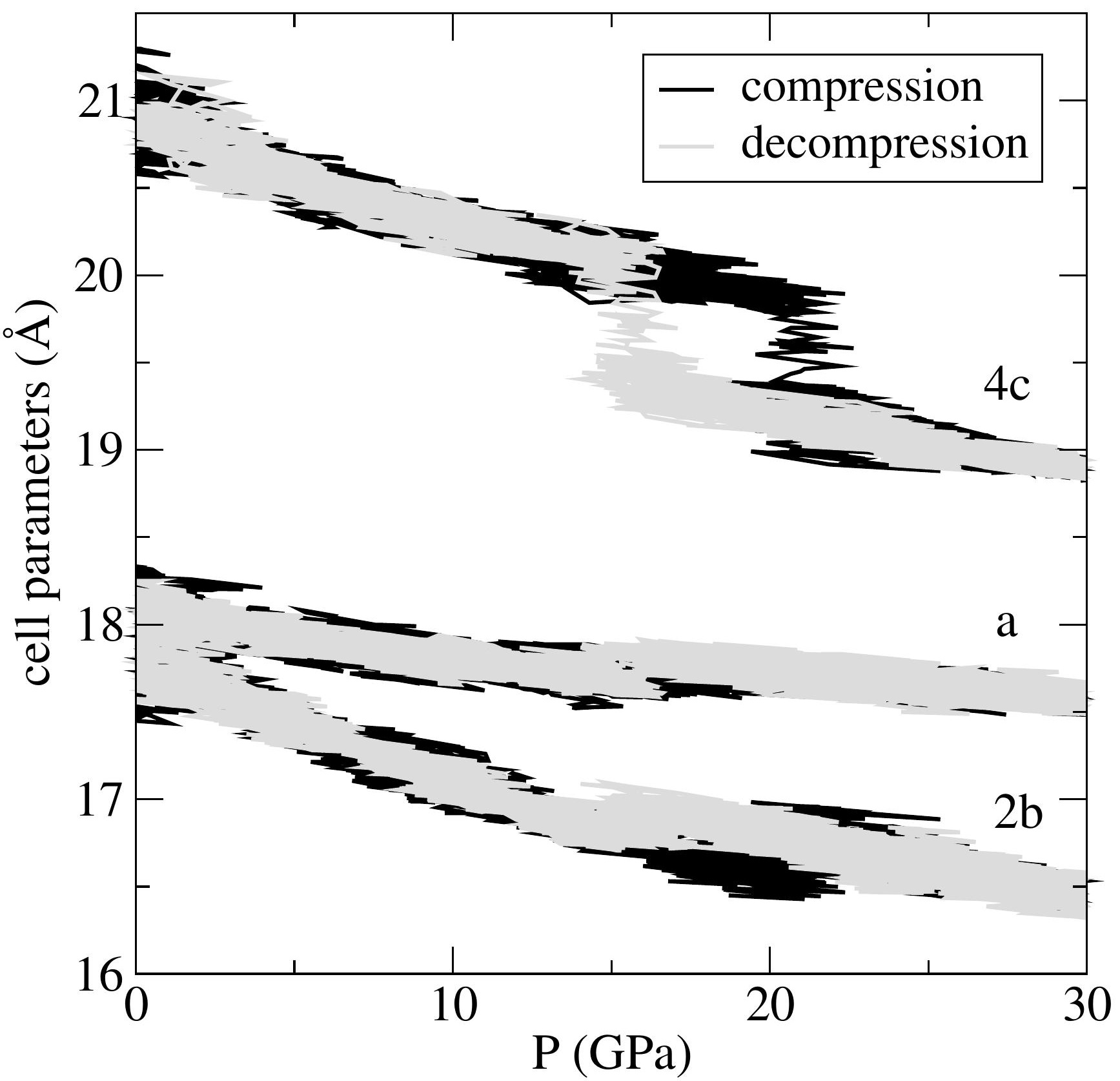




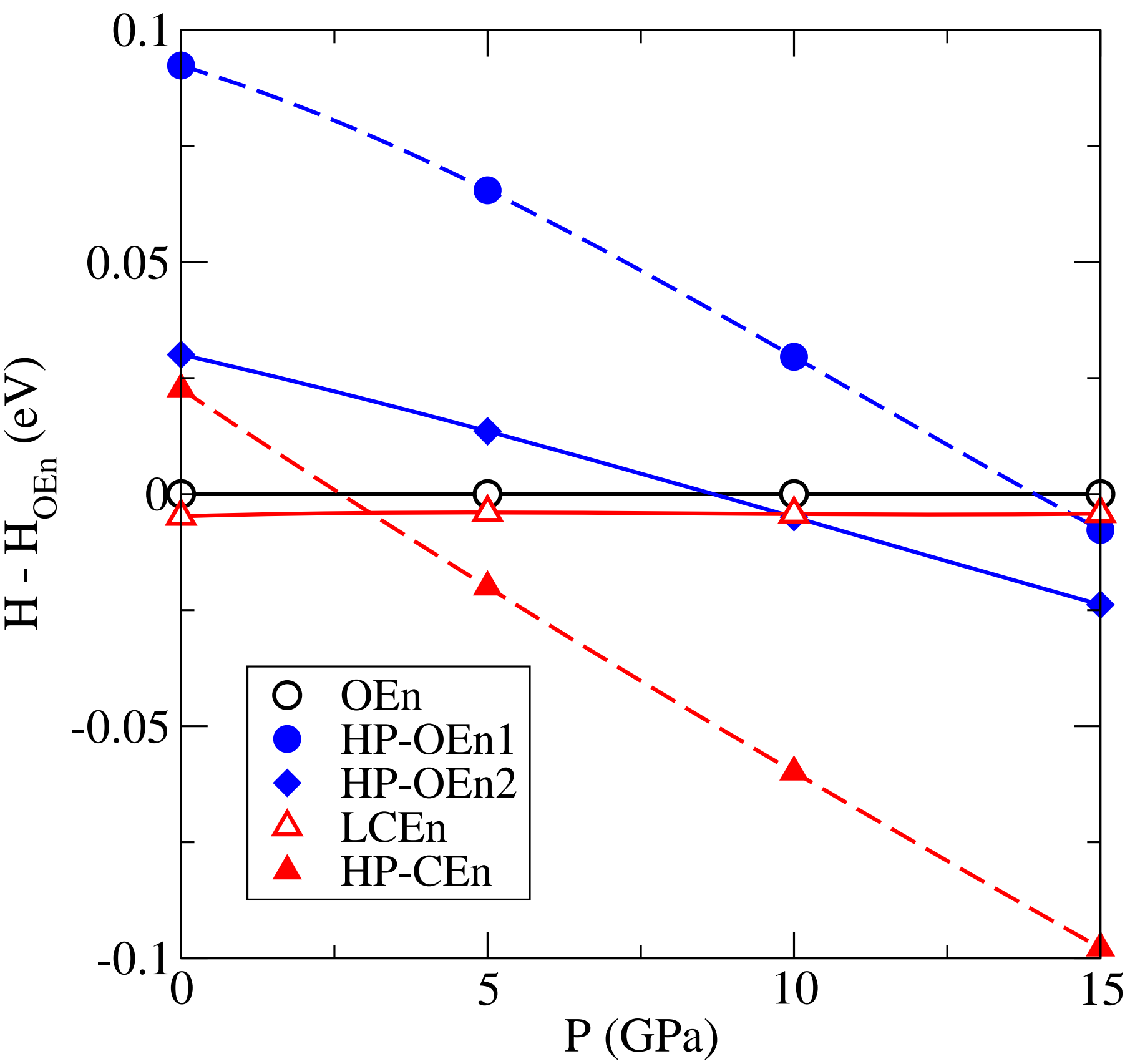




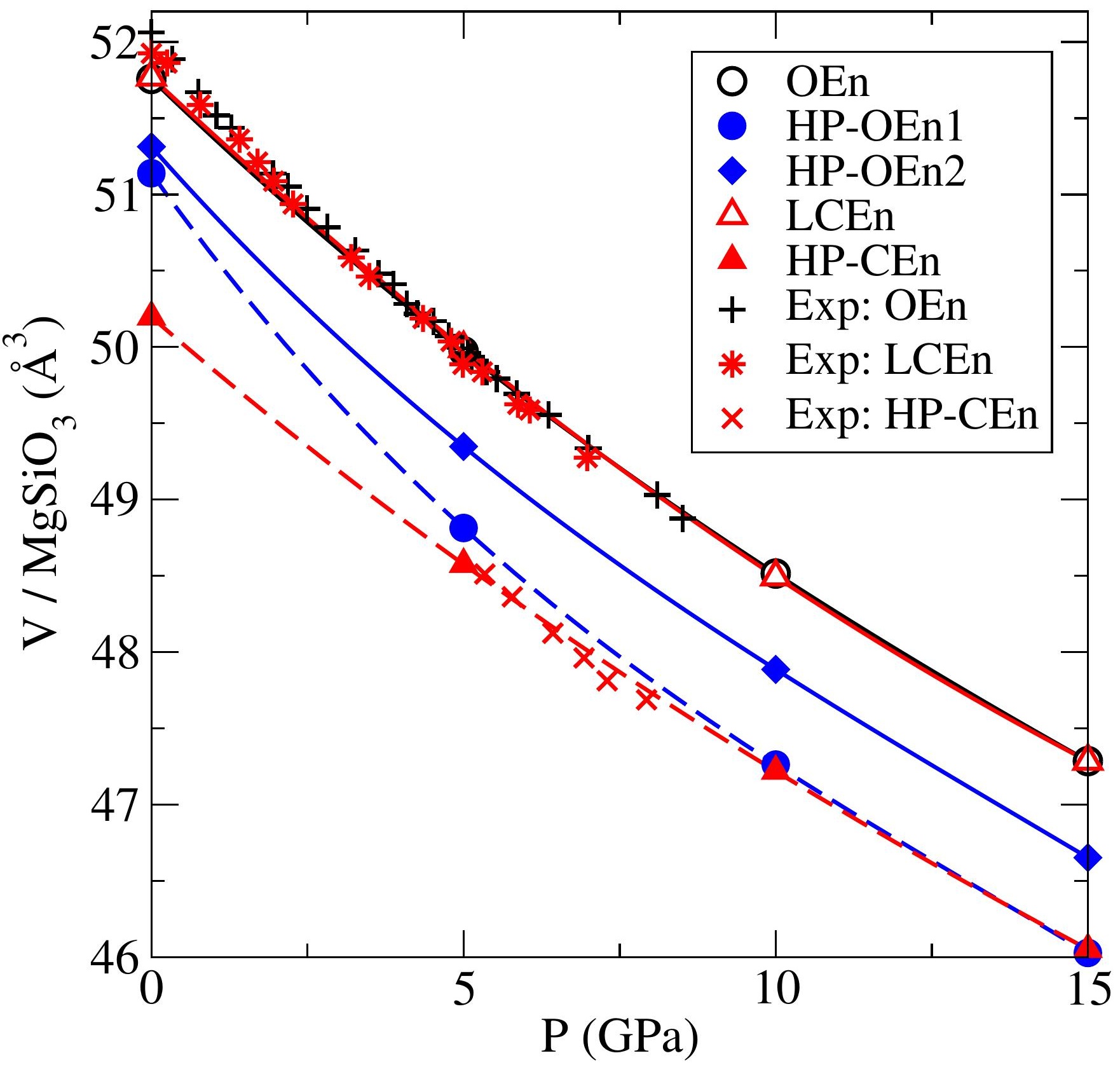


intensity (a.u.)

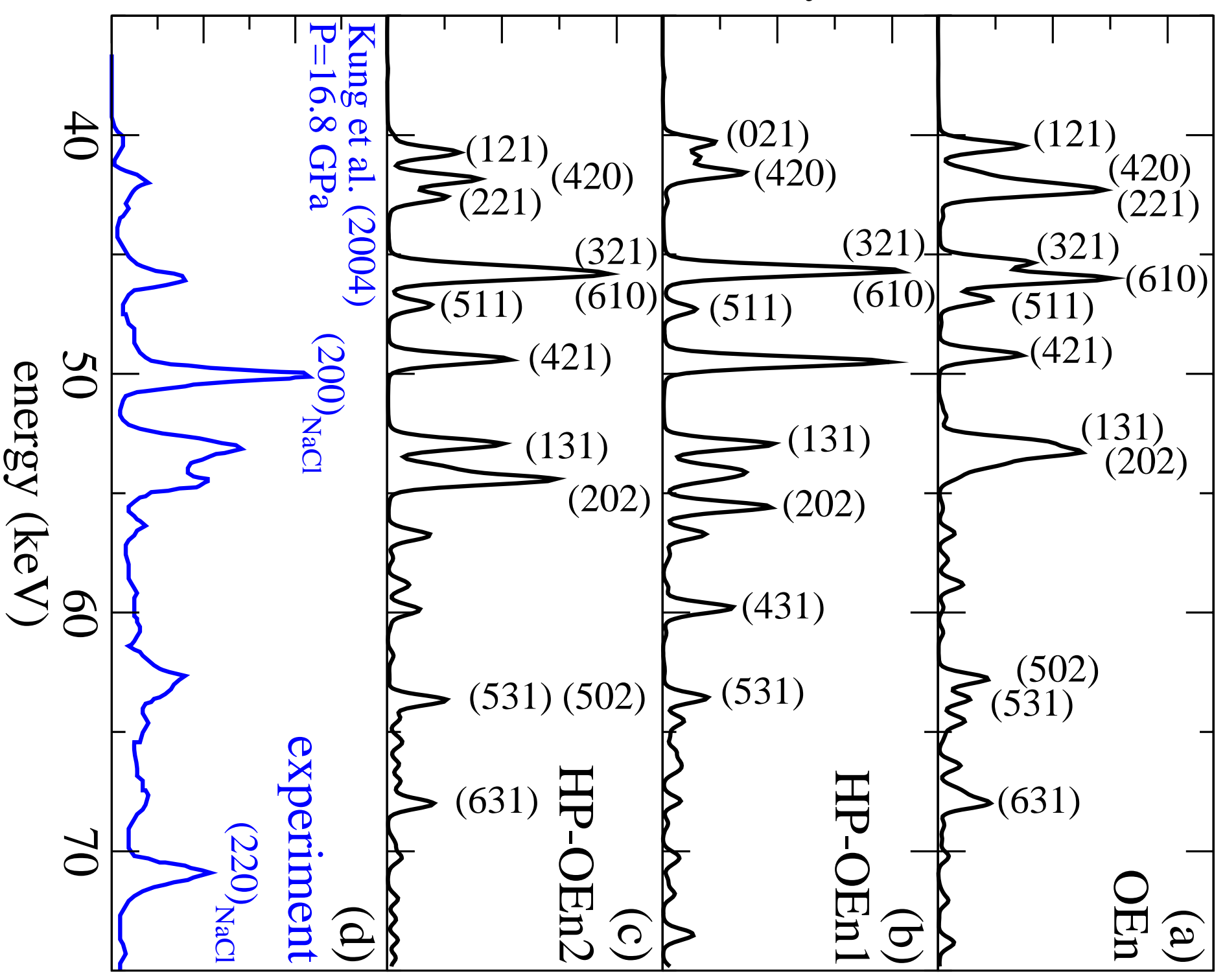


OEn

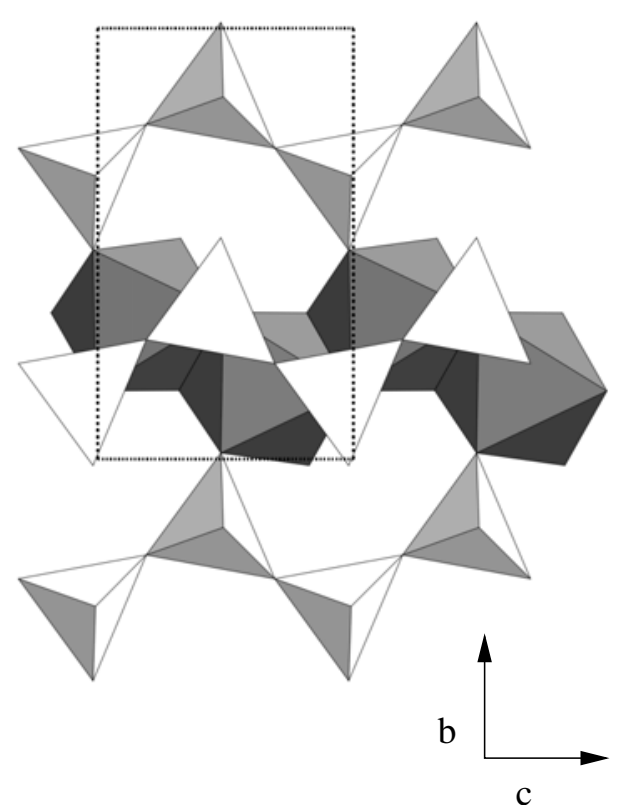

HP-OEn1

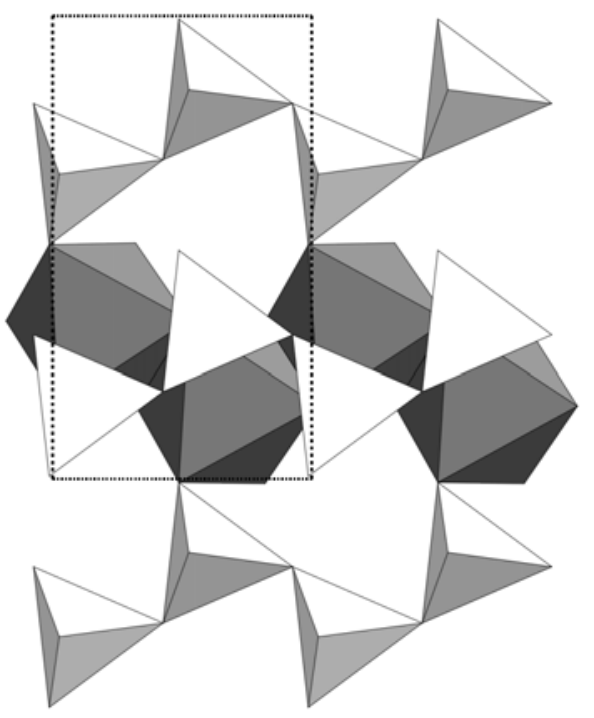

HP-OEn2

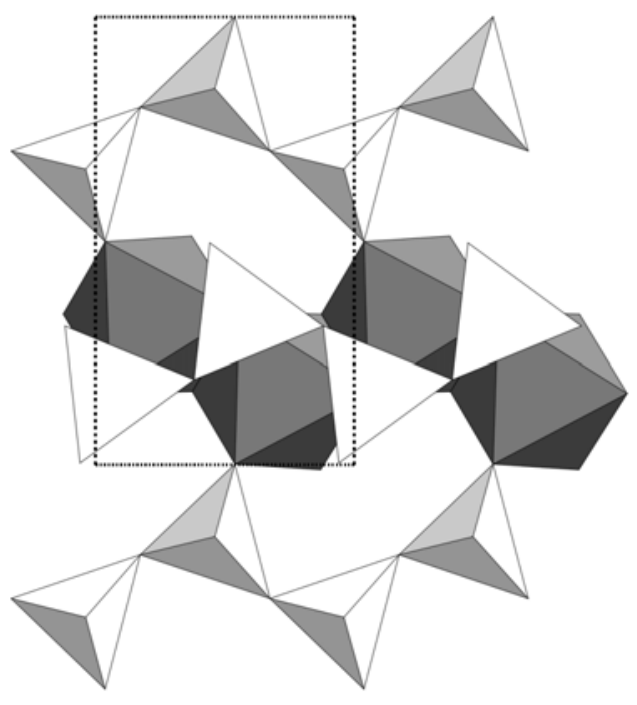

